\title{
MAMIFEROS DE LOS ALREDEDORES DEL SANTUARIO TABACONAS-NAMBALLE, CAJAMARCA. LISTA PRELIMINAR
}

\author{
JUAN JOSÉ RODRÍGUEZ G. (*) \\ Asociación de Ecología y Conservación \\ (ECCO-PERU)
}

\begin{abstract}
RESUMEN
Visitas a cuatro caseríos que constituyen alrededores nor este del Santuario Nacional Tabaconas-Namballe (San Ignacio, Cajamarca) entre junio y julio de 1994, permitieron registrar un total de 26 especies de la fauna mastozoológica pertenecientes a 7 órdenes, (Marsupialia, Chiroptera, Primates, Xenarthra, Rodentia, Carnivora y Artiodactyla ) y 13 familias. Se elabora el primer listado de mamíferos para la zona.

PALABRAS CLAVE: Mamíferos, santuario, Tabaconas-Namballe.
\end{abstract}

\section{SUMMARY}

In 1994, during June and July, visits to four hamlets around (nort eastern) National Sanctuary Tabaconas-Namballe in San Ignacio, Cajamarca, permited to register 26 species of the mammals fauna that belong to 7 orders ( Marsupialia, Chiroptera, Primates, Xenarthra, Rodentia, Carnivora, and Artiodactyla ) and 13 families. The first list of mammals for this zone has been elaborated.

KEY WORD: Mammals, santuary, Tabaconas-Namballe.

\section{INTRODUCCION}

El Santuario Nacional Tabaconas-Namballe, con una extensión de 29,500 hectáreas está ubicado en el Departamento de Cajamarca, Provincia de San Ignacio, Distritos de Tabaconas y Namballe (Figura 1.). Es particularmente importante porque alberga a especies en situación rara o en vías de extinción, tales como Tapirus pinchaque (tapir de altura) y Tremarctos ornatus (oso andino) y que actualmente están sujetos a una fuerte presión de caza. Se encuentra también una muestra de los bosques de Podocarpus sp. (Romerillo) que a pesar de ser declarado intangible, es extraído por los pobladores. Se realiza un primer inventario de diversidad de la mastofauna en los alrededores del Santuario. Estos resultados serán utilizados, posteriormente, para sugerir la ampliación de los límites de éste y permitir que las especies en vías de extinción tengan una mayor distribución y mejorar su status.

Las localidades visitadas fueron El Quebradón (1,200 m.s.n.m.), La Unión (1,100 m.s.n.m.), Pueblo Libre (1,560 m.s.n.m) y un pequeño caserío ubicado a 2 kilómetros al oeste del anterior al que llamaremos Pueblo Libre $2(1,700$ m.s.n.m.)

\section{MATERIAL Y METODOS}

Se utilizaron trampas de golpe «Víctor» para el registro de roedores y marsupiales pequeños y redes de neblina para registros de quirópteros. Las trampas de golpe fueron colocadas en cinco transectos de $100 \mathrm{~m}$. con 10 trampas en cada transecto y separadas entre sí por una distancia de $10 \mathrm{~m}$. en zonas que comprendían áreas cultivadas, intervenidas (Zonas que los pobladores locales utilizan para vivienda o extracción de madera) y bosques. Las redes de neblina ( $12 \times 2 \mathrm{~m}$.) fueron colocadas en cafetales, frutales y bosques en lugares estratégicos del vuelo de estos individuos.

Los especímenes colectados fueron preparados como pieles de estudio, los cráneos separados del cuerpo y conservados en alcohol $70 \%$ y la carcasa preservada en formol $10 \%$. Se tomaron datos sobre longitud total, longitud de la cola, longitud de la pata, longitud de la oreja, peso, sexo y estado reproductivo (Nagorsen y col. 1980).

Los registros de mamíferos medianos y grandes se realizaron por observación directa, detección de huellas o marcas en los árboles (Tremarctos ornatus) y por la obtención de cráneos y pieles que fueron proporcionados por los pobladores.

\section{RESULTADOS Y DISCUSION}

Se aplicaron cinco días de muestreo efectivo en cada una de las cuatro zonas estudiadas, empleando trampas de

(*) Nueva dirección: Museo de Historia Natural, Universidad Ricardo Palma, Santiago de Surco, Lima-Perú. 
golpe para roedores pequeños, redes de neblina para la captura de quirópteros y observaciones directas para mamíferos medianos y grandes.

En los caseríos de Pueblo Libre y Pueblo Libre 2 (ubicados en los límites del Santuario) se pudo registrar la mayor diversidad de especies, estando éstas localidades menos intervenidas que El Quebradón y La Unión que se encuentran a 15 y 10 kilómetros respectivamente del Santuario.

La especie más común para Marsupialia es Marmosops noctivagus «Shanda» encontrándose en los cuatro lugares visitados. Las descripciones, para este trabajo, de Oligoryzomys destructor, Oligoryzomys albigularis y Oryzomys albigularis necesitan confirmación (Patton 1994, com. per.).

Los quirópteros fueron identificados utilizando la clave de Murciélagos de Venezuela (Linares, 1987) y Ascorra (com. per.). La identificación de los otros grupos se confirmó en Hemmons y Feer. (1990).

Según entrevistas a los pobladores, se determinan especies dañinas para la agricultura local, entre éstas Dasyprocta sp. (Dasyproctidae, Rodentia) «Chosco», y Agouti paca (Agoutidae, Rodentia) «Majas», que se alimentan de plátanos (Musaceae), vituca (Araceae) y yuca(Euforbiaceae); tambiénMustela frenata (Mustelidae, Carnivora) «Chucurillo», dañino para los animales menores de granja como cuyes, gallinas y pollos, $y$ Didelphis marsupialis (Didelphidae, Marsupialia) «muca» que ingresa a diferentes cultivos y se alimentan también de aves de corral.

Se elabora el primer listado de mamíferos para los alrededores nor este del Santuario Tabaconas-Namballe con un total de 7 órdenes, 13 familias y 26 especies que se indican en la tabla 1. Es de esperar que la diversidad de mamíferos de la región sea mayor, para ello se requieren continuar las evaluaciones en diferentes épocas estacionales.

Tabla 1. Lista de mamíferos registrados en los caseríos de El Quebradón, La Unión, Pueblo Libre y Pueblo Libre 2. Alrededores nor-este del Santuario Nacional TabaconasNamballe, San Ignacio, Cajamarca, Perú.

\section{Marsupialia \\ Didelphidae \\ Didelphis marsupialis \\ Marmosops noctivagus}

\section{Chiroptera}

Phyllostomidae

Carollia spp.

Desmodus rotundus
Artibeus jamaicensis

Artibeus lituratus

Uroderma bilobatum

Sturnira lilium

Sturnira magna

Chrotopterus auritus

Lonchorina aurita

Glossophaga soricina

\section{Primates \\ Cebidae \\ Cebus albifrons \\ Alouatta seniculus}

\section{Xenarthra}

Dasypodidae

Dasypus novemcinctus

\section{Rodentia}

Sciuridae

Sciurus spadiceus

Muridae

Oligoryzomys albigularis

Oligoryzomys destructor

Oryzomys albigularis

Dasyproctidae

Dasyprocta sp.

Agoutidae

Agouti paca

\section{Carnivora}

Mustelidae

Mustella frenata

Eira barbara

Ursidae

Tremarctos ornatus

Procyonidae

Nasua nasua

\section{Artiodactyla \\ Cervidae \\ Mazama americana.}

\section{REFERENCIAS BIBLIOGRAFICAS}

HEMMONS, L.; F. FEER 1990. Neotropical Rainforest Mammals. A Field Guide. The University of Chicago Press. 281p.

LINARES,O. 1987. Murciélagos de Venezuela. Cuadernos Lagoven. $120 \mathrm{p}$.

NAGORSEN, D., R.PETERSON. 1980. Mammal Collectors' Manual. Life Sciences Miscellaneus Publications. Royal Ontario Museum. Toronto, Canada. 79p. 